\title{
UM LEVANTAMENTO DA PRESENÇA DA DISCIPLINA DE DIDÁTICA DA MATEMÁTICA EM CURSOS DE LICENCIATURA A DISTÂNCIA EM INSTITUIÇÕES PÚBLICAS BRASILEIRAS
}

\author{
Claudia Maria Witt ${ }^{1}$ \\ Maria Lucia Panossian²
}

\begin{abstract}
RESUMO
O presente artigo apresenta os resultados da pesquisa cujo objetivo foi realizar um levantamento de cursos de Licenciatura em Matemática a Distância que contemplem a disciplina Didática da Matemática em suas grades curriculares. Para atingir este objetivo foi realizada pesquisa no site do Ministério da Educação na intenção de identificar quantos cursos e quais instituições públicas brasileiras ofertam cursos nesta modalidade de ensino. Identificadas as instituições, foram coletados de seus sites o plano de curso, a grade curricular, a ementa do curso e a ementa da disciplina Didática da Matemática para a realização de análise documental. Pela análise dos documentos que foram possíveis de serem coletados, é percebido que a disciplina Didática da Matemática não é contemplada em todos os cursos de Licenciatura em Matemática a Distância. Em alguns cursos, seus conhecimentos são abordados em outras disciplinas pedagógicas. A partir dos resultados desta análise pretende-se discutir a necessidade e importância que os conhecimentos em Didática da Matemática trazem para a formação do professor. Os dados aqui apresentados são um recorte do projeto de Mestrado em andamento que tem como questão desencadeadora o que caracteriza a disciplina Didática da Matemática nos cursos de Licenciatura a Distância.
\end{abstract}

Palavras-chave: Licenciatura em matemática a distância. Didática da matemática. Formação inicial docente.

A SURVEY OF THE PRESENCE OF THE DISCIPLINE OF MATHEMATICS IN DISTANCE LEARNING COURSES IN BRAZILIAN PUBLIC INSTITUTIONS

\section{ABSTRACT}

This study presents the results of the research that has had as goal to map the courses of Distance Learning Degree in Mathematics that consider the subject Didactic of Mathematics in their curriculum charts. To reach this goal, a research in the site of Ministry of Education was necessary with the intention to identify the quantity of courses and in which Brazilian Public Institutions offer this course in their education system. As soon as the institutions had been detected, the Course Plan, the curriculum chart, the course summary and the summary of the subject Didactic of Mathematics were collected to be realized the documental analysis. According this analysis was possible to detect that the subject Didactic of Mathematic has not been offering in all courses of Distance Learning Degree in Mathematics. In some courses, its knowledges are focus on other pedagogical subjects. From the results of this study, there is the intention to discuss the necessity and the importance that the knowledge in didactic in mathematics brings to the teachers training. The presented data is a cutting of a Master Degree in progress as an unleashed question that distinguishes the subject Didactic of Mathematics in Distance Learning Degree Courses.

Keywords: Degree in learning distance mathematics. Didactics of mathematics. Initial teacher training.

Recebido em: $17 / 9 / 2018$

Aceito em: $11 / 12 / 2019$

\footnotetext{
${ }^{1}$ Mestranda do Programa de Pós-Graduação em Educação em Ciência e em Matemática - PPGECM da Universidade Federal do Paraná UFPR Curitiba. http://lattes.cnpq.br/1839942991833865. https://orcid.org/0000-0003-4132-5912. claudiamariaw@gmail.com

2 Doutora em Educação pela USP - SP. Professora do Programa de Pós-Graduação em Educação em Ciências e em Matemática - PPGECM da Universidade Federal do Paraná - UFPR Curitiba. http://lattes.cnpq.br/3750368511104051. https://orcid.org/0000-0001-5847-4485. mlpanossian@utfpr.edu.br
} 
Muitas são as preocupações dos pesquisadores no que diz respeito à formação docente e após a publicação das Diretrizes Curriculares Nacionais para a formação do Professor da Educação Básica, em nível superior, curso de Licenciatura, de Graduação Plena (BRASIL, 2001a) e da publicação das Diretrizes Curriculares Nacionais para os cursos de Matemática, Bacharelado e Licenciatura (BRASIL, 2001b), intensificaram-se as discussões acerca das reformulações das licenciaturas em Matemática quanto aos novos modelos de formação docente (NACARATO; PAIVA, 2013). Estes novos modelos ampliaram-se com os avanços científicos e com a grande ebulição tecnológica, aprimorando os modelos existentes e trazendo novas possibilidades à formação de professores.

A Licenciatura em Matemática a Distância adquiriu espaço neste contexto de mudanças e se configurou como uma nova possibilidade de formação. A necessidade de formar profissionais para atuarem nas escolas de Educação Básica, promovendo o desenvolvimento social, cultural e econômico da população, norteou o processo de constituição e desenvolvimento dos cursos de Formação de Professores a Distância. Estes, no entanto, precisam ser cuidadosamente "desenhados" e gerenciados para que sejam eficientes para os aprendizes, levando-se em conta a materialidade histórica dos sujeitos envolvidos neste processo para que não se reduzam "a um rol de conceitos a ser incorporado, sem atentar se tais conceitos são de fato significativos à sua formação" (PESCE, 2009, p. 134).

Neste sentido é que se propôs a realização desse levantamento. Por meio dele torna-se possível conhecer a realidade da Licenciatura em Matemática a Distância oferecida em instituições públicas brasileiras. Este levantamento possibilitou identificar as instituições credenciadas e com autorização de funcionamento pelo Ministério da Educação no período de abril a agosto de 2017.

A análise de como se estruturam tais cursos revela a vastidão de possibilidades que estes oferecem para se compreender o processo de formação do professor de Matemática. Desta maneira, tornou-se necessário delimitar o olhar para os cursos analisados e, assim, optou-se por identificar a presença da disciplina Didática da Matemática ${ }^{3}$ nos cursos de Licenciatura em Matemática a Distância.

Considerando que os conhecimentos da disciplina Didática da Matemática oferecidos ao professor em formação oportunizam sua instrumentalização para exercer sua atividade docente e para o seu desenvolvimento como sujeito é que surge a necessidade de se discutir a presença da disciplina nos cursos de Licenciatura a Distância no cenário brasileiro, almejando contribuir com discussões que aprimorem os processos de formação de professores de Matemática.

\section{MATERIAIS E MÉTODOS}

Para a realização desta pesquisa buscou-se identificar quantas e quais instituições públicas brasileiras estão credenciadas e têm autorização do Ministério da Educação (MEC) para ofertarem a Licenciatura em Matemática na modalidade a Distância. Este

Quando as expressões "didática" e "didática da matemática" referirem-se a conhecimento científico, utilizar-se-ão iniciais minúsculas e quando se referirem às disciplinas de "Didática" e de "Didática da Matemática", adotar-se-ão as iniciais maiúsculas. 
levantamento foi realizado durante no período de abril a agosto de 2017, por meio do link <http://emec.mec.gov.br>, no qual foi possível encontrar 43 instituições públicas ofertando 43 cursos de Licenciatura em Matemática na modalidade a Distância e 1 instituição oferecendo o curso de Licenciatura em Ciências Naturais e Matemática na mesma modalidade, contemplando quase todos os Estados brasileiros. Nesta busca não apareceram registros destes cursos nos Estados do Espírito Santo (ES), Amazonas (AM) e Rondônia (RO), assim como no Distrito Federal (DF) e nem a oferta de polos de outra instituição. Estes dados obtidos estão dispostos no quadro a seguir:

Quadro 1 - Instituições públicas brasileiras x Licenciatura em Matemática a distância

\begin{tabular}{|c|c|c|c|}
\hline Universidade/Instituição - Estado & Sigla & Qt. Polos & Localização Polos \\
\hline Universidade Federal de Pelotas - RS & UFPel & 29 & 1 (SC), 1 (PR) e 27 (RS) \\
\hline Universidade Federal do Rio Grande do Sul - RS & UFRGS & 15 & 1 (MS) e $14(\mathrm{RS})$ \\
\hline Universidade Federal do Rio Grande - RS & FURG & 5 & 5 (RS) \\
\hline Universidade Federal de Santa Catarina - SC & UFSC & 28 & $11(\mathrm{MA})$ e $17(\mathrm{SC})$ \\
\hline Universidade Estadual de Ponta Grossa - PR & UEPG & 28 & $28(P R)$ \\
\hline $\begin{array}{l}\text { Instituto Federal de Educação, Ciência e Tecnologia do } \\
\text { Triângulo Mineiro - MG }\end{array}$ & IFTM & 8 & $4(\mathrm{MG})$ e $4(\mathrm{SP})$ \\
\hline Universidade Federal de Ouro Preto - MG & UFOP & 17 & $13(\mathrm{MG})$ e 4 (SP) \\
\hline Universidade Federal de São João Del Rei - MG & UFSJ & 12 & $5(M G)$ e 7 (SP) \\
\hline Universidade Federal do Estado do Rio de Janeiro - RJ & UNIRIO & 5 & $5(\mathrm{RJ})$ \\
\hline Universidade Federal Fluminense - RJ & UFF & 18 & $18(\mathrm{RJ})$ \\
\hline $\begin{array}{l}\text { Instituto Federal de Educação, Ciência e Tecnologia do } \\
\text { Norte de Minas Gerais - MG }\end{array}$ & IFNMG & 2 & 2 (MG) \\
\hline Universidade Federal de Juiz de Fora - MG & UFJF & 12 & 12 (MG) \\
\hline Universidade Federal de Minas Gerais - MG & UFMG & 8 & 8 (MG) \\
\hline Universidade Federal de Viçosa - MG & UFV & 4 & 4 (MG) \\
\hline $\begin{array}{l}\text { Universidade Federal dos Vales do Jequitinhonha e } \\
\text { Mucuri - MG }\end{array}$ & UFVJM & 7 & 7 (MG) \\
\hline $\begin{array}{l}\text { Fundação Universidade Federal de Mato Grosso do Sul } \\
\text { - MS }\end{array}$ & UFMS & 8 & 8 (MS) \\
\hline Fundação Universidade Federal do Mato Grosso - MT & UFMT & 6 & $6(\mathrm{MT})$ \\
\hline $\begin{array}{l}\text { Fundação Universidade Federal do Mato Grosso - MT } \\
\left({ }^{*}\right)\end{array}$ & UFMT & 12 & 12 (MT) \\
\hline Universidade Federal de Goiás - GO & UFG & 5 & $5(\mathrm{GO})$ \\
\hline Fundação Universidade Federal do Tocantins - TO & UFT & 14 & $14(\mathrm{TO})$ \\
\hline Universidade do Tocantins - TO & UNITINS & 8 & $8(\mathrm{TO})$ \\
\hline Universidade Federal do Acre - AC & UFAC & 9 & $9(A C)$ \\
\hline $\begin{array}{l}\text { Instituto Federal de Educação, Ciência e Tecnologia do } \\
\text { Pará - PA }\end{array}$ & IFPA & 9 & $1(P A)$ e 8 (RR) \\
\hline Universidade Federal do Pará - PA & UFPA & 10 & $1(\mathrm{AP})$ e 9 (PA) \\
\hline Universidade do Estado do Pará - PA & UEPA & 1 & $1(\mathrm{PA})$ \\
\hline Universidade Federal de Roraima - RR & UFRR & 9 & $9(\mathrm{RR})$ \\
\hline Universidade Federal do Amapá - AP & UNIFAP & 5 & $5(\mathrm{AP})$ \\
\hline Universidade Federal do Maranhão - MA & UFMA & 9 & 9 (MA) \\
\hline Universidade Estadual do Piauí - PI & UESPI & 8 & $8(\mathrm{PI})$ \\
\hline Universidade Federal do Piauí - PI & UFPI & 28 & 1 (BA) e 27 (PI) \\
\hline $\begin{array}{l}\text { Instituto Federal de Educação, Ciência e Tecnologia do } \\
\text { Ceará - CE }\end{array}$ & IFCE & 13 & $13(\mathrm{CE})$ \\
\hline
\end{tabular}




\begin{tabular}{|l|c|c|c|}
\hline Universidade Estadual do Ceará - CE & UECE & 6 & 6 (CE) \\
\hline Universidade Federal do Ceará - CE & UFC & 12 & 12 (CE) \\
\hline $\begin{array}{l}\text { Instituto Federal de Educação, Ciência e Tecnologia de } \\
\text { Pernambuco - PE }\end{array}$ & IFPE & 8 & $\begin{array}{c}1 \text { (AL), } 1 \text { (BA) e } 6 \text { (PE) } \\
6 \text { (PE) }\end{array}$ \\
\hline Universidade Federal de Pernambuco - PE & UFPE & 6 & 6 (PE) \\
\hline Universidade do Estado da Bahia - BA & UNEB & 41 & 41 (BA) \\
\hline Universidade Estadual do Sudoeste da Bahia - BA & UESB & 5 & 5 (BA) \\
\hline Universidade Federal da Bahia - BA & UFBA & 18 & 18 (BA) \\
\hline Universidade Federal do Recôncavo da Bahia - BA & UFRB & 1 & 1 (BA) \\
\hline Universidade Federal da Paraíba - PB & UFPB & 21 & 2 (BA) e 19 (PB) \\
\hline Universidade Federal do Rio Grande do Norte - RN & UFRN & 10 & 10 (RN) \\
\hline Universidade Federal Rural do Semi-Árido - RN & UFERSA & 4 & 4 (RN) \\
\hline Universidade Federal de Alagoas - AL & UFAL & 3 & 3 (AL) \\
\hline Universidade Federal de Sergipe - SE & UFS & 14 & 14 \\
\hline
\end{tabular}

* Curso de Licenciatura em Ciências Naturais e Matemática.

Fonte: Dados da Pesquisa, Ministério da Educação (http://emec.mec.gov.br). Acesso: abr. 2017. Organizado pelas autoras.

Pelos dados coletados percebe-se que algumas instituições oferecem o curso também em polos localizados em outros Estados brasileiros, buscando ampliar a oferta de vagas para que mais pessoas tenham acesso à formação docente.

Diante de tal cenário encontrado é possível afirmar que a Licenciatura em Matemática a Distância é uma realidade concretizada e que não cabe mais discutir, pois vem de longa data, uma vez que no final da década de 70 surgiu a primeira experiência de EaD nos cursos superiores. O Parecer CNE/CES no 564/2015 justificava este fato, quando em 1972, o conselheiro Newton Sucupira, do Conselho Federal de Educação (CFE), em visita à Open University, na Inglaterra, deu início ao processo defendendo a criação de uma universidade aberta, pois segundo ele, "além de ampliar 'as oportunidades de acesso à educação superior [...] é um processo de educação permanente em nível universitário'" (BARRETO, 2001 apud BRASIL, 2015b, p. 5).

O desafio que se impõe atualmente, porém, é pensar quais necessidades emergem para que o processo de apropriação do conhecimento e de desenvolvimento humano do licenciando nesta modalidade se efetive. $E$, buscando ilustrar o cenário nacional encontrado na pesquisa, construiu-se o mapa seguinte com o objetivo de facilitar a localização de cada uma das instituições brasileiras que disponibilizam o curso de Licenciatura em Matemática a Distância. 
Figura 1 - Instituições Públicas Brasileiras x Licenciatura em Matemática a Distância

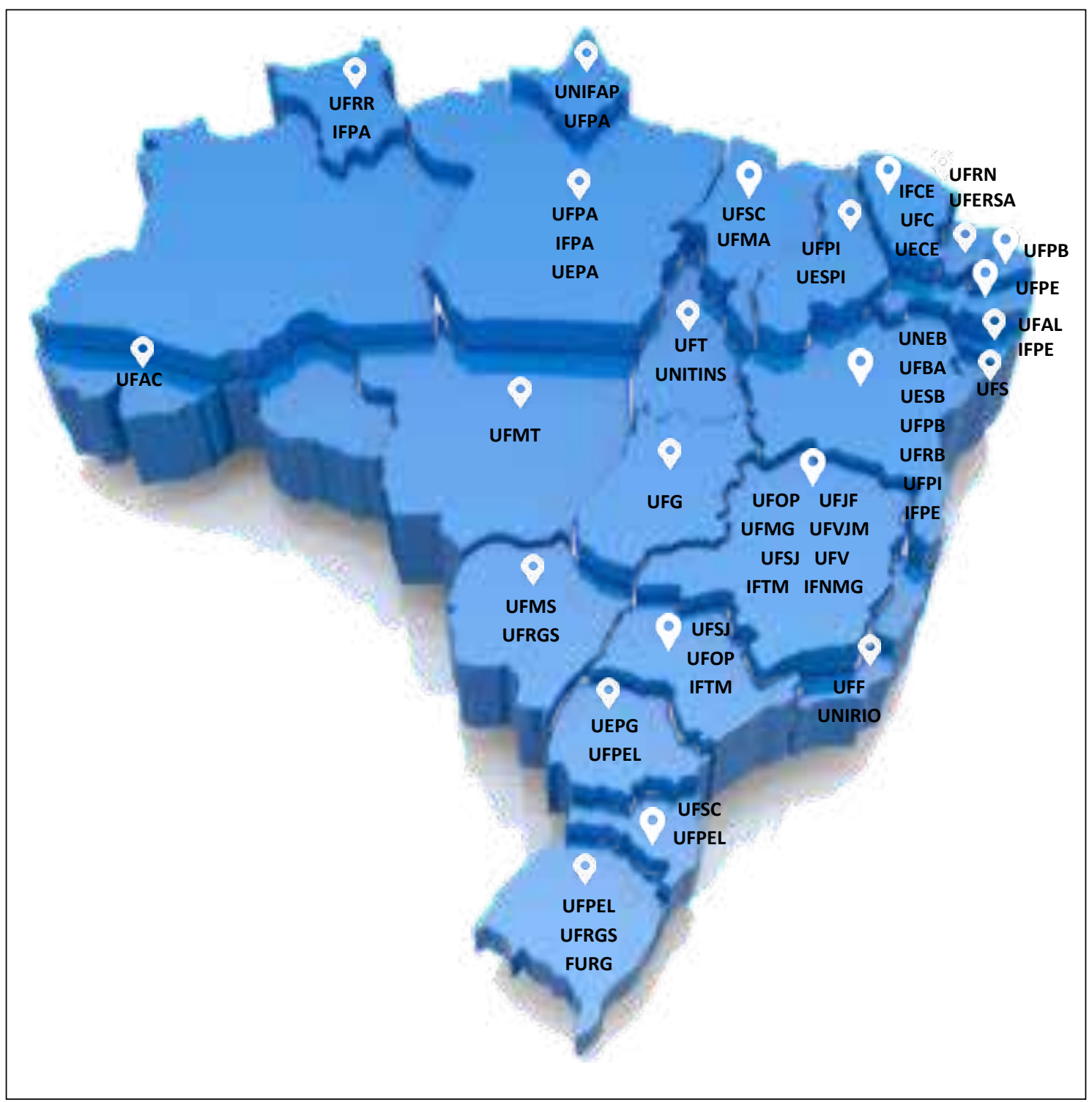

Fonte: Dados da Pesquisa, Ministério da Educação, Abril de 2017. Organizado pelas autoras.

Identificadas e localizadas geograficamente as instituições públicas brasileiras que oferecem o curso de Licenciatura em Matemática a Distância, o passo seguinte foi investigar no site de cada uma das instituições catalogadas pelo MEC os links para acesso à página do curso de Licenciatura em Matemática a Distância.

Nesta etapa, o objetivo foi encontrar seus planos de curso, grades curriculares, ementas do curso e da disciplina Didática da Matemática e bibliografias sugeridas para se poder identificar a presença da disciplina Didática da Matemática nestes cursos.

Foi constatado que algumas Instituições de Ensino Superior (IESs) não disponibilizam acesso ao curso para coleta de documentos sem que se esteja matriculado nele. Foram então coletados os documentos das instituições que os disponibilizam publicamente.

Estes documentos obtidos estão relacionados no quadro seguinte, sendo identificados por "sim", foi possível coletá-lo, ou "não", não foi possível coletá-lo: 
Quadro 2 - Documentos Coletados dos Cursos de Licenciatura em Matemática a Distância

\begin{tabular}{|c|c|c|c|}
\hline Universidade/Instituição - Estado & $\begin{array}{c}\text { Plano de } \\
\text { Curso }\end{array}$ & $\begin{array}{c}\text { Grade } \\
\text { Curricular }\end{array}$ & Ementa \\
\hline Universidade Federal de Pelotas - UFPel - RS & Não & Sim & Não \\
\hline Universidade Federal do Rio Grande do Sul - UFRGS - RS & Não & Não & Não \\
\hline Universidade Federal do Rio Grande - FURG - RS & Não & Não & Não \\
\hline Universidade Federal de Santa Catarina - UFSC - SC & Sim & Sim & Sim \\
\hline Universidade Estadual de Ponta Grossa - UEPG - PR & Não & Sim & Sim \\
\hline Instituto Federal de Educação, Ciência e Tecnologia do Triângulo Mineiro - IFTM - MG & Sim & Sim & Sim \\
\hline Universidade Federal de Ouro Preto - UFOP - MG & Sim & Sim & Sim \\
\hline Universidade Federal de São João Del Rei - UFSJ - MG & Sim & Sim & Sim \\
\hline Universidade Federal do Estado do Rio de Janeiro - UNIRIO - RJ & Sim & Sim & Sim \\
\hline Universidade Federal Fluminense - UFF -RJ & Não & Sim & Sim \\
\hline Instituto Federal de Educação, Ciência e Tecnologia do Norte de Minas Gerais - IFNMG - MG & Não & Não & Não \\
\hline Universidade Federal de Juiz de Fora - UFJF - MG & Não & Não & Não \\
\hline Universidade Federal de Minas Gerais - UFMG - MG & Não & Sim & Não \\
\hline Universidade Federal de Viçosa - UFV - MG & Não & Sim & Não \\
\hline Universidade Federal dos Vales do Jequitinhonha e Mucuri - UFVJM - MG & Sim & Sim & Sim \\
\hline Fundação Universidade Federal de Mato Grosso do Sul - UFMS - MS & Não & Não & Não \\
\hline Fundação Universidade Federal do Mato Grosso - UFMT-MT & Sim & Sim & Sim \\
\hline Fundação Universidade Federal do Mato Grosso - UFMT - MT (*) & Sim & $\operatorname{Sim}$ & Sim \\
\hline Universidade Federal de Goiás - UFG - GO & Não & Não & Não \\
\hline Fundação Universidade Federal do Tocantins - UFT - TO & Sim & $\operatorname{Sim}$ & Sim \\
\hline Universidade do Tocantins - UNITINS - TO & Não & Sim & Não \\
\hline Universidade Federal do Acre - UFAC - AC & Não & Não & Não \\
\hline Instituto Federal de Educação, Ciência e Tecnologia do Pará - IFPA - PA & Não & Não & Não \\
\hline Universidade Federal do Pará - UFPA - PA & Sim & Sim & Sim \\
\hline Universidade do Estado do Pará - UEPA - PA & Não & Não & Não \\
\hline Universidade Federal de Roraima - UFRR - RR & Sim & Sim & Sim \\
\hline Universidade Federal do Amapá - UNIFAP - AP & Não & Não & Não \\
\hline Universidade Federal do Maranhão - UFMA - MA & Sim & Sim & Sim \\
\hline Universidade Estadual do Piauí - UESPI - PI & Não & Não & Não \\
\hline Universidade Federal do Piauí - UFPI - PI & Não & Não & Não \\
\hline Instituto Federal de Educação, Ciência e Tecnologia do Ceará - IFCE - CE & Não & Não & Não \\
\hline Universidade Estadual do Ceará - UECE - CE & Não & Sim & Não \\
\hline Universidade Federal do Ceará - UFC - CE & Sim & Sim & Sim \\
\hline Instituto Federal de Educação, Ciência e Tecnologia de Pernambuco - IFPE - PE & Sim & Sim & Sim \\
\hline Universidade Federal de Pernambuco -- UFPE - PE & Não & Não & Não \\
\hline Universidade do Estado da Bahia - UNEB - BA & Não & Não & Não \\
\hline Universidade Estadual do Sudoeste da Bahia - UESB - BA & Não & Não & Não \\
\hline Universidade Federal da Bahia - UFBA - BA & Não & Não & Não \\
\hline Universidade Federal do Recôncavo da Bahia - UFRB - BA & Sim & Sim & Sim \\
\hline Universidade Federal da Paraíba - UFPB - PB & Sim & Sim & Sim \\
\hline Universidade Federal do Rio Grande do Norte - UFRN - RN & Sim & Sim & Sim \\
\hline Universidade Federal Rural do Semi-Árido - UFERSA - RN & Sim & Sim & Sim \\
\hline Universidade Federal de Alagoas - UFAL - AL & Sim & Sim & Sim \\
\hline Universidade Federal de Sergipe - UFS - SE & Não & Não & Não \\
\hline
\end{tabular}

* Curso de Licenciatura em Ciências Naturais e Matemática.

Fonte: Ministério da Educação (http://emec.mec.gov.br). Acesso: jul. 2017. Organizado pelas autoras. 
Com os documentos obtidos foi realizada a análise buscando identificar em seus planos de curso os objetivos para a formação do professor de Matemática nesta modalidade de ensino. Pelas grades curriculares, a análise buscou a presença da disciplina de Didática da Matemática como componente curricular da formação, bem como o momento e a carga horária dedicada à disciplina. Estes dados revelam que a disciplina de Didática da Matemática não é abordada em todos os cursos de Licenciatura em Matemática a Distância. Com esta constatação, a análise passou a ser realizada nos cursos que apresentam em suas grades curriculares a disciplina Didática da Matemática, sendo realizada por meio das ementas propostas pelo curso e pela disciplina em questão.

\section{RESULTADOS E DISCUSSÕES}

Conforme a tabela exposta anteriormente, foi possível coletar 19 planos de curso, 26 grades curriculares e 21 ementas de curso.

Pelas 26 grades curriculares encontradas pode-se constatar a presença das disciplinas de Didática e de Didática da Matemática nos cursos de Licenciatura em Matemática a Distância das instituições públicas brasileiras.

Tem-se, conforme o quadro a seguir, que 18 cursos oferecem na formação inicial os conhecimentos da disciplina Didática Geral. A disciplina de Didática da Matemática aparece em 8 cursos. Em 4 deles aborda-se ambas as disciplinas e em outros 4 há somente a presença da disciplina Didática da Matemática. Estas informações podem ser verificadas no quadro a seguir.

Quadro 3 - Disciplinas na Grade Curricular dos Cursos de Licenciatura em Matemática a Distância

\begin{tabular}{|c|c|c|}
\hline \multirow[b]{2}{*}{ Universidade/Instituição - Estado } & \multicolumn{2}{|c|}{ Disciplina } \\
\hline & $\begin{array}{c}\text { Didática } \\
\text { Geral }\end{array}$ & $\begin{array}{l}\text { Didática da } \\
\text { Matemática }\end{array}$ \\
\hline Universidade Federal de Pelotas - UFPel - RS & Não & Não \\
\hline Universidade Federal de Santa Catarina - UFSC - SC & Sim & Sim \\
\hline Universidade Estadual de Ponta Grossa - UEPG - PR & Sim & Não \\
\hline Instituto Federal de Educação, Ciência e Tecnologia do Triângulo Mineiro - IFTM - SP & Sim & Não \\
\hline Universidade Federal de Ouro Preto - UFOP - SP & Não & Sim \\
\hline Universidade Federal de São João Del Rei - UFSJ - SP & Não & Sim \\
\hline Universidade Federal do Estado do Rio de Janeiro - UNIRIO - RJ & Sim & Não \\
\hline Universidade Federal Fluminense - UFF - RJ & Sim & Não \\
\hline Universidade Federal de Minas Gerais - UFMG - MG & Sim & Não \\
\hline Universidade Federal de Viçosa - UFV - MG & Não & Não \\
\hline Universidade Federal dos Vales do Jequitinhonha e Mucuri - UFVJM - MG & Sim & Não \\
\hline Fundação Universidade Federal do Mato Grosso - UFMT - MT & Sim & Sim \\
\hline Fundação Universidade Federal do Mato Grosso - UFMT - MT (*) & Não & Não \\
\hline Fundação Universidade Federal do Tocantins - UFT - TO & Sim & Não \\
\hline Universidade do Tocantins - UNITINS - TO & Sim & Não \\
\hline Universidade Federal do Pará - UFPA - PA & Não & Não \\
\hline Universidade Federal de Roraima - UFRR - RR & Sim & Não \\
\hline Universidade Federal do Maranhão - UFMA - MA & Sim & Não \\
\hline Universidade Estadual do Ceará - UECE - CE & Sim & Não \\
\hline Universidade Federal do Ceará - UFC - CE & Sim & Não \\
\hline Instituto Federal de Educação, Ciência e Tecnologia de Pernambuco - IFPE - PE & Sim & Sim \\
\hline Universidade Federal do Recôncavo da Bahia - UFRB - BA & Não & Sim \\
\hline Universidade Federal da Paraíba - UFPB - PB & Sim & Não \\
\hline
\end{tabular}




\begin{tabular}{|l|c|c|}
\hline Universidade Federal do Rio Grande do Norte - UFRN - RN & Sim & Não \\
\hline Universidade Federal Rural do Semi-Árido - UFERSA - RN & Sim & Sim \\
\hline Universidade Federal de Alagoas - UFAL - AL & Não & Sim \\
\hline
\end{tabular}

* Curso de Licenciatura em Ciências Naturais e Matemática a Distância.

Fonte: Site da Instituição com oferta do curso. Acesso: ago. 2017. Organizado pela autora.

Após a verificação de que a disciplina de Didática da Matemática dentro das 26 grades curriculares encontradas é contemplada em 8 cursos de Licenciatura em Matemática a Distância, buscou-se pela sua denominação dentro de cada curso, sua carga horária e o período da formação no qual ela é abordada.

Encontramos denominações para a disciplina como "Iniciação à Pesquisa em Didática da Matemática". Neste curso sua carga horária é de cem horas, sem a presença da Prática como Componente Curricular e aparecendo no oitavo período da formação.

Em outra instituição denomina-se "Didática da Matemática", tendo carga horária de 60 horas, não contemplando a Prática como Componente Curricular e ocorrendo no oitavo período do curso.

Em outra, está intitulada também como "Didática da Matemática", totalizando carga horária de 72 horas sem a Prática como Componente Curricular e aparece no terceiro semestre da formação.

Noutro curso recebe a denominação de "Didática para o Ensino de Matemática", disponibilizando uma carga horária de 32 horas "Teóricas" mais 32 horas de Prática como Componente Curricular, totalizando 64 horas de estudo e ocorrendo no quinto período do curso.

Outra instituição a denomina de "Didática da Matemática", contando com carga horária "Teórica" de 60 horas sem a Prática como Componente Curricular e aparecendo no sexto período do curso.

Em outra instituição é contemplada no quarto período da formação, sendo denominada de "Prática de Ensino V - Didática da Matemática", com carga horária de 60 horas sem a presença da Prática como Componente Curricular.

Denominada de "Didática da Matemática" em outra instituição, conta com uma carga horária total de 60 horas definida como "Prática" no oitavo período do curso.

E na oitava instituição que apresenta em sua grade curricular a disciplina, ela recebe o nome de "Fundamentos da Didática da Matemática", tendo carga horária de 68 horas "Teóricas" definidas no quinto semestre da formação.

Partindo desta observação foi diagnosticada a variação de carga horária assim como o momento do curso dedicado ao oferecimento dos conhecimentos em didática da matemática. Percebe-se que cada curso organiza-se de acordo com suas necessidades e concepções e que a inclusão dos conhecimentos em Didática da Matemática no processo de formação docente em algumas destas instituições fica relegada ao final do curso. Varizo (2013) atenta para isso, observando que a disciplina Didática da Matemática "não pode [...] ser mais uma disciplina isolada, ministrada no final do curso de formação de professores" (p. 56), pois os conhecimentos teóricos que ela oferece ao professor em formação sustentarão sua ação em sala de aula. 
Durante esta análise percebeu-se que uma das instituições que apresenta em sua grade curricular a disciplina Didática da Matemática divide sua carga horária em Teórica e em Prática como Componente Curricular. Convém então destacar que a Resolução no 2, de 2015, em seu artigo 13, §1으, parágrafo I, prevê "400 (quatrocentas) horas de prática como componente curricular, distribuídas ao longo do processo formativo", e seu $\S 3$ o determina que "Deverá ser garantida, ao longo do processo, efetiva e concomitante relação entre teoria e prática, ambas fornecendo elementos básicos para o desenvolvimento dos conhecimentos e habilidades necessários à docência".

Esta divisão proposta pelo curso indica que a dimensão do conhecimento do professor presente nos momentos de reflexão sobre a atividade profissional e a dimensão teórica do curso ganham espaço com a inserção da Prática como Componente Curricular, pois seus elementos constitutivos, suas exigências legais e sua articulação no Projeto Pedagógico da instituição formadora, como estabelece a legislação e citado por Figueiredo e Costa (2015) definem que, "essas atividades práticas devem transcender o estágio e ter como finalidade a promoção da articulação de diferentes práticas por meio de uma perspectiva interdisciplinar" e ainda, "não poderá ficar isolada, ou seja, restrita ao estágio, sem conexão com o resto do curso" (p. 122).

Como o interesse nesta pesquisa recai sobre a presença da disciplina de Didática da Matemática, seus conhecimentos e sua abordagem como disciplina na grade curricular da Licenciatura a distância, a análise dos dados exigiu um olhar para as ementas da disciplina destes 8 cursos. Considera-se que muito do que se concebe sobre os seus conhecimentos venham de uma ideologia construída culturalmente para se dar "coesão aos indivíduos em seus papéis, em suas funções e em suas relações sociais" (HARNECKER, 1983, p. 101), e que necessitam manifestar-se "em todos os atos e gestos dos indivíduos [ao ponto de] ser indiscernível de sua 'experiência vivida' e, por isso, toda análise imediata do 'vivido' está profundamente marcada pela ação da ideologia” (Ibidem, p. 102).

Para identificar as 8 instituições públicas brasileiras que contemplam a disciplina Didática da Matemática em suas grades curriculares, construiu-se o quadro a seguir e o mapa na sequência (Figura 2), que localiza geograficamente estes cursos no Brasil.

Quadro 4 - Cursos que Contemplam a Disciplina de Didática da Matemática

\begin{tabular}{|l|c|}
\hline \multicolumn{1}{|c|}{ Universidade/Instituição - Estado } & $\begin{array}{c}\text { Didática da } \\
\text { Matemática }\end{array}$ \\
\hline Universidade Federal de Santa Catarina - UFSC - SC & Sim \\
\hline Universidade Federal de Ouro Preto - UFOP - MG & Sim \\
\hline Universidade Federal de São João Del Rei - UFSJ - MG & Sim \\
\hline Fundação Universidade Federal do Mato Grosso - UFMT - MT & Sim \\
\hline Instituto Federal de Educação, Ciência e Tecnologia de Pernambuco - IFPE - PE & Sim \\
\hline Universidade Federal do Recôncavo da Bahia - UFRB - BA & Sim \\
\hline Universidade Federal Rural do Semi-Árido - UFERSA - RN & Sim \\
\hline Universidade Federal de Alagoas - UFAL - AL & Sim \\
\hline
\end{tabular}

Fonte: Site da instituição com oferta da disciplina no curso. Acesso: ago. 2017. Organizado pelas autoras. 
Figura 2 - Instituições Públicas Brasileiras x Disciplina de Didática da Matemática

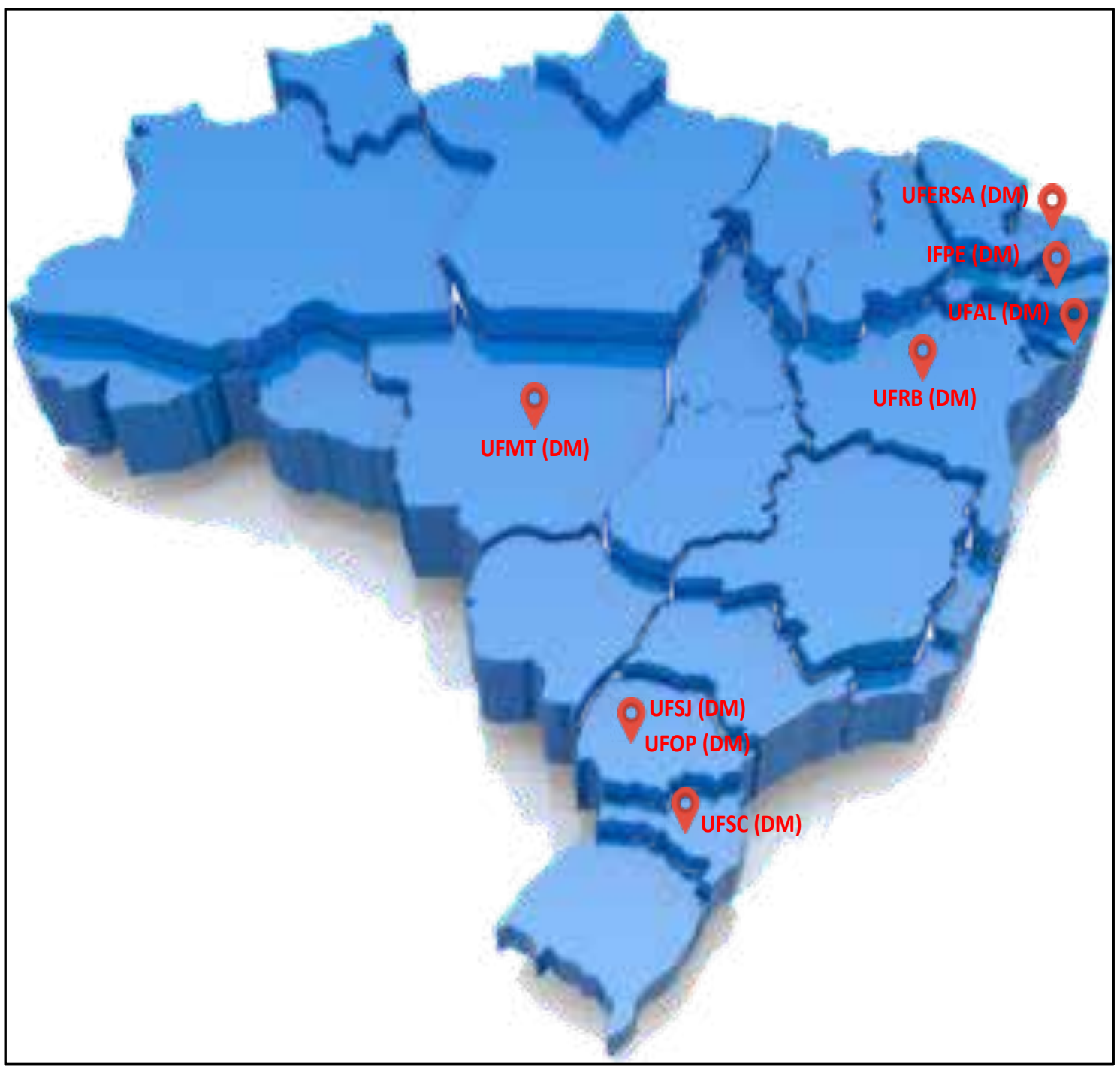

Fonte: Dados da Pesquisa, Ministério da Educação. Agosto de 2017. Organizado pelas autoras.

Destes 8 cursos, identificados como A, B, C, D, E, F, G e H, que apresentam em sua grade curricular a disciplina de Didática da Matemática, voltou-se o olhar para suas ementas. A primeira intenção foi identificar os objetivos propostos para a disciplina de Didática da Matemática na formação do professor de Matemática na Licenciatura a distância, tendo como entendimento que "os mesmos elementos desempenham papel diferente conforme seja a organização do todo e o lugar que nele ocupam" (HARNECKER, 1983 , p. 87). Nesse sentido, o que se entende e o que se pratica como didática da matemática no processo de formação para a docência manifesta-se na ação do professor quando em atuação.

Sabe-se que a ementa é o documento que tem como finalidade expor as propostas de cada componente curricular e quanto mais informações ela disponibilizar, mais claros ficam os objetivos para a formação docente. Nem todas as ementas, contudo, apresentam-se assim. Algumas explicitam de modo geral o que propõe a disciplina, como segue: 
Construção de uma prática pedagógica de matemática que se desenvolva dentro dos princípios didáticos: Objetivos, conteúdos, formas organizativas do ensino, métodos, meios de ensino e sistema de avaliação da aprendizagem, na direção de um processo ensino; aprendizagem (sic) de matemática adequado aos alunos. De um modo coerente e criativo em que conteúdos, objetivos e métodos, meios, formas e avaliação se integrem de acordo com a intencionalidade educativa do professor de matemática, da escola e da sociedade (INSTITUIÇÃO B).

Estrutura e funcionamento do sistema didático como modelo teórico, Obstáculos Epistemológicos; Dialética Ferramenta-Objeto; Engenharia didática; Campos Conceituais; Construção do conhecimento matemático (INSTITUIÇÃO E).

Introdução à Didática da Matemática; o sistema didático; a sala de aula de matemática e os diferentes fenômenos didáticos; transposição didática (externa e interna); teoria das situações didáticas; teoria dos campos conceituais; erros e obstáculos (INSTITUIÇÂO F).

O compromisso político do educador no ensino da matemática. A relação teoria-prática na construção da matemática e no trabalho pedagógico do professor. A séria busca do lúdico na matemática (INSTITUIÇÃO G).

Reflexões sobre o que é Matemática, a matemática que se aprende e a que se ensina, os objetivos de seu ensino no Ensino Fundamental (5a a $8^{a}$ séries) e Médio. Apresentação de diversos métodos (resolução de problemas, uso da História da Matemática, uso de materiais didáticos e recursos tecnológicos, modelagem matemática, dentre outros) para o ensino de Matemática com vistas ao planejamento de unidades didáticas. Implementação por meio de aulas simuladas das aulas preparadas. A temática das aulas simuladas abrangerá os campos da Aritmética, Álgebra, Geometria, Tratamento da Informação, Princípios de Combinatória e Probabilidade, Conjuntos Numéricos, Análise Combinatória, Probabilidade, Estatística e Matemática Financeira. Planejamento de projetos inter-disciplinares. Análise, avaliação e escolha de livros didáticos para o Ensino Fundamental e Médio (INSTITUIÇÃO H).

Quando se observam os objetivos para a disciplina de Didática da Matemática nos outros 3 cursos, identifica-se uma intenção mais voltada ao educador matemático. Assim, eles se propõe a:

[...] fornecer elementos teóricos e metodológicos que contribuam para o exercício do ser professor pesquisador na sua rotina de trabalho [oportunizando-lhe] o conhecimento de noções fundamentais das teorias da educação matemática (INSTITUIÇÃO A).

Analisar as principais concepções referentes à educação e à formação do educador matemático; Refletir sobre diferentes abordagens do ensino de matemática; Estudar, desenvolver e projetar uma concepção educacional de ensino de matemática; Analisar e discutir diferentes recursos didáticos no ensino da Matemática; Compreender os elementos que constituem a organização do processo de ensino e aprendizagem matemática: planejamento, ensino, avaliação, seus significados e práticas (INSTITUIÇÃO C). 
Apresentar as metodologias necessárias para a apresentação de uma boa aula. Entender os mecanismos para o bom aprendizado do aluno; Organizar-se para que a aula seja a melhor possível com o máximo de aproveitamento; Entender a necessidade para de uma boa apresentação; Entender os fundamentos da didática aplicando o conhecimento matemático (INSTITUIÇÃO D).

Neste olhar para as ementas da disciplina também revela-se quais autores aparecem mais destacados. $O$ quadro a seguir mostra quais aparecem com maior frequência na lista de bibliografia básica ou complementar das instituições e o número de vezes citados:

Quadro 5 - Autores Utilizados na Disciplina Didática da Matemática e Número de Vezes que Aparece nas Ementas das Instituições Analisadas

\begin{tabular}{|c|c|}
\hline BIBLIOGRAFIA BÁSICA E/OU COMPLEMENTAR & CITADO EM \\
\hline 1. ALMOULOUD, S. A. Fundamentos da Didática da Matemática. Curitiba: UFPR, 2007. & Duas (2) \\
\hline 2. ALVES, F. R. V. Didática da Matemática. UAB/IFCE, Fortaleza, 2011. & Uma (1) \\
\hline 3. ANTUNES, C. Matemática e Didática. Editora Vozes, 2010. & Uma (1) \\
\hline $\begin{array}{l}\text { 4. COMENIUS (1997) DIDÁTICA MAGNA - Editora Martins Fontes - Primeira Edição: Ou- } \\
\text { tubro de 1997. - Capítulo } 11 \text { (Páginas: } 106 \text { a 108). }\end{array}$ & Duas (2) \\
\hline $\begin{array}{l}\text { 5. DANTE, L.R. Didática da resolução de problemas de matemática. São Paulo: Ática, } \\
\text { 1989. }\end{array}$ & Três (3) \\
\hline $\begin{array}{l}\text { 6. D’AMORE, B. Epistemologia e didática da Matemática. São Paulo: Escrituras, 2005. Tí- } \\
\text { tulo do original: Le basi filosofiche, pedagogiche, epistemologiche e concettuali della } \\
\text { Didatica dela matemática. }\end{array}$ & Uma (1) \\
\hline $\begin{array}{l}\text { 7. D’AMORE, Bruno. Elementos de didática da matemática. São Paulo: Livraria da Física, } \\
2007\end{array}$ & Duas (2) \\
\hline 8. NETO, E. R. Didática da Matemática. Coleção Educação, 12. ed. Editora Atica, 1991. & Uma (1) \\
\hline $\begin{array}{l}\text { 9. PAIS, Luiz Carlos. Didática da matemática: Uma análise da influência francesa. } 2 \text { ed. } \\
\text { Belo Horizonte: Autêntica.128 p. }\end{array}$ & Cinco (5) \\
\hline 10. PARRA, C. Didática da Matemática: Reflexões Psicopedagógicas. Editora Penso, 1996. & Uma (1) \\
\hline $\begin{array}{l}\text { 11. PARRA, C; SAIZ, I. (org.). Didática da Matemática: reflexões psicopedagógicas. Trad. } \\
\text { Juan Acuña Llorens. Porto Alegre: Artes Médicas, } 1996 .\end{array}$ & Três (3) \\
\hline
\end{tabular}

Fonte: Os autores.

Convém considerar, pelo que foi encontrado, que nem todos os cursos incluem a disciplina de Didática da Matemática no currículo da formação docente a Distância. Assim sendo, torna-se necessário olhar para a ementa dos cursos que não apresentam a disciplina Didática da Matemática em sua grade curricular, buscando identificar em qual componente os seus conhecimentos estão sendo abordados durante a formação do licenciando. Esta análise possibilitou identificar que uma provável abordagem de seus conhecimentos possa estar acontecendo em disciplinas intituladas "Instrumentação para o ensino de Matemática", identificada em 3 cursos (identificados como Instituições I, J e K), "Matemática e Educação", em 1 curso (identificado como Instituição L) e "Metodologia para o Ensino de Matemática" em 1 curso (com a identificação de Instituição $M$ ).

Nestas disciplinas percebe-se uma aproximação a questões mais específicas aos conhecimentos voltados ao ensino da Matemática. 
$\mathrm{Na}$ instituição I as disciplinas de "Instrumentação do Ensino da Geometria" e "Instrumentação do Ensino da Aritmética e Álgebra", com carga horária, cada uma, de 75 horas, constantes no sétimo e oitavo período do curso, aproximam seus conhecimentos aos de didática da matemática. Percebe-se aproximação quando busca

[...] relação com a organização formal do pensamento; confecção e utilização de materiais pedagógicos adequados ao ensino-aprendizagem de conteúdos geométricos relacionados à Análise, Álgebra e outras Ciência [e] uma análise crítica, do ponto de vista lógico, cognitivo e didático de textos de Matemática e de Educação Matemática; relações elementares entre a Matemática e outras disciplinas/Ciências, os quais possam instrumentalizar o ensino da matemática elementar (EMENTA DO CURSO DA INSTITUIÇÃO I).

$\mathrm{Na}$ instituição J, uma aproximação pode estar acontecendo nas disciplinas de "Instrumentação para o Ensino da Matemática I e II", em que se pretende:

[...] reflexões sobre o que é Matemática, a matemática que se aprende e a que se ensina, os objetivos de seu ensino [...], diversos métodos (resolução de problemas, uso da História da Matemática, uso de materiais didáticos e recursos tecnológicos, modelagem matemática, dentre outros) para o ensino de Matemática com vistas ao planejamento de unidades didáticas. Análise, utilização e construção de materiais didáticos (EMENTA DO CURSO DA INSTITUIÇÃO J).

E na instituição $\mathrm{K}$ os conhecimentos em didática da matemática são abordados nas disciplinas de "Instrumentação para o Ensino de Matemática I, II, III, IV e V", discutindo assuntos como:

O professor de Matemática no atual contexto social: identidade, formação e atuação profissional. Domínios de conhecimento necessários ao professor de Matemática: conhecimento matemático, conhecimento sobre a aprendizagem matemática e conhecimento da didática da Matemática. Matemática e Educação Matemática. Diretrizes Curriculares Nacionais para o Ensino da Matemática. Inter-relação dos conteúdos matemáticos que compõem as disciplinas do primeiro/segundo semestre do curso e destes com a Educação Básica (EMENTA DO CURSO DA INSTITUIÇÃO K).

O curso da Instituição L aproxima os conhecimentos em didática da matemática no componente curricular "Matemática e Educação". Entende-se tal fato quando sua ementa define:

Estudo de tendências do ensino de Matemática: investigação matemática, modelagem, formulação e resolução de problemas e etnomatemática. Análise dos elementos e fundamentos do processo de ensino e aprendizagem de matemática nesse contexto: concepção de ensino, concepção de aprendizagem e papéis de alunos e professores. Prática de ensino como componente curricular (EMENTA DO CURSO DA INSTITUIÇÃO L).

Nesta instituição tais conhecimentos acontecem na disciplina intitulada "Matemática e Educação IV", inserida no sexto período da formação, com carga horária de 60 horas, definida como Prática como Componente Curricular. 
$\mathrm{Na}$ instituição $\mathrm{M}$ tem-se uma aproximação aos conhecimentos em didática da matemática na disciplina intitulada "Metodologia para o Ensino da Matemática I", ocorrendo no quarto semestre da formação e com carga horária de 90 horas. Sua ementa define a abordagem de:

Fundamentos teóricos do ensino da matemática, concepções filosóficas no ensino da matemática, currículo, competências e habilidades, o perfil do professor de matemática, da teoria à prática: resolução de problemas, etnomatemática modelagem matemática, jogos e desafios matemáticos, calculadora: mocinha ou vilã? Softwares matemáticos, (re)significação matemática, laboratório de matemática, avaliação em matemática e análise do livro didático e paradidático (EMENTA DO CURSO DA INSTITUIÇÃO M).

É preciso estar atento, no entanto, se a disciplina de Didática da Matemática não está sendo confundida com Metodologia de Ensino, a qual, segundo Costa (2013, p. 184), é "o conjunto de métodos e técnicas que são utilizados a fim de que o processo ensino-aprendizagem se realize com êxito". Para que isso não aconteça é preciso aceitar que a Didática deve ser vista "como uma matéria de integração" que se nutre "dos conhecimentos e práticas desenvolvidos nas metodologias específicas e nas outras ciências pedagógicas para formular generalizações em torno dos conhecimentos e tarefas docentes comuns e fundamentais ao processo de ensino" (LIBÂNEO, 2013, p. 9). Assim, a Didática está conectada com as metodologias específicas, as práticas de ensino e o estágio, pois conforme Libâneo (2013, p. 12) "todas as matérias do currículo partem, incluem e levam à prática de ensino [e] em particular, há uma fecundação mútua entre Didática e as metodologias específicas, não se concebendo uma sem as outras".

Necessário também levar em consideração que, para orientar sua ação pedagógica, o professor necessita de conhecimentos didáticos-pedagógicos que sustentem sua ação. E é neste campo de conhecimentos que se situa a didática da matemática. Considerada muitas vezes como mais uma das disciplinas pedagógicas pelas quais o futuro professor precisa passar para conquistar o tão almejado diploma de licenciado, levando-se em conta que os conhecimentos em Didática Geral já seriam suficientes para instrumentalizar o futuro professor para sua atividade docente.

Desta maneira, acredita-se na necessidade de se discutir a formação inicial docente no contexto da Licenciatura em Matemática a Distância, voltando o olhar para a disciplina Didática da Matemática pela contribuição que seus conhecimentos trazem ao licenciando quando incluída na grade curricular dos cursos de Licenciatura em Matemática.

Imbuídos da certeza das possibilidades que os conhecimentos em Didática da Matemática trazem para a formação docente é que se defende a importância da sua abordagem durante o processo de formação, pois ela é responsável por fornecer conhecimentos teóricos e práticos que permitem

[...] que o professor de Matemática veja seu papel em um contexto mais amplo, assim como "educador matemático". Ser um educador matemático é muito mais do que um simples "transmissor de conteúdo", assumindo o seu verdadeiro papel como educador, onde a crítica, o questionamento permanente, a autodetermi- 
nação e a independência nas formas de atuação e especialmente no pensamento também são encorajados através da Matemática (ESPINOSA, 2011, p. 9, tradução nossa).

\section{CONSIDERAÇÕES FINAIS}

A realização deste levantamento de cursos de Licenciatura em Matemática a Distância oferecido por instituições públicas brasileiras fornece um panorama de como tem se estruturados os cursos desta modalidade, revelando informações importantes quanto à disciplina de Didática da Matemática.

Nesse sentido, o que se entende e o que se pratica como didática da matemática no processo de formação para a docência manifesta-se na ação do professor quando em atuação. Acredita-se que um dos desafios da formação docente a distância seja assegurar ao licenciando conhecimentos capazes de proporcionar-lhe condições de organizar o ensino da matemática de modo que promova o desenvolvimento cognitivo de seus futuros alunos.

Considera-se necessário então discutir a importância da presença da disciplina Didática da Matemática na formação docente, a qual oportuniza uma melhor compreensão da atividade pedagógica do professor, para que ela não venha a se caracterizar como uma "prática vazia, uma prática pela prática" (VARIZO, 2013, p. 56), possibilitando "variar o discurso acadêmico dos professores no ensino dos conteúdos fundamentais da Matemática" (Ibidem).

\section{REFERÊNCIAS}

BRASIL. Ministério da Educação. Conselho Nacional de Educação. Parecer CNE/CP 9/2001. 2001a. Disponível em: http://portal.mec.gov.br/cne/arquivos/pdf/009.pdf. Acesso em: jul. 2018.

BRASIL. Ministério da Educação. Conselho Nacional de Educação. Parecer CNE/CES 1.302/2001. 2001b. Disponível em: http://portal.mec.gov.br/cne/arquivos/pdf/CES13022.pdf. Acesso em: jul. 2018.

BRASIL. Ministério da Educação. Conselho Nacional de Educação. Conselho Pleno. Resolução no 2, de 1o de julho de 2015. 2015a. Disponível em: http://portal.mec.gov.br/docman/agosto-2017-pdf/70431-res-cne-cp-002-03072015-pdf/file Acesso em: ago. 2017.

BRASIL. Ministério da Educação. Conselho Nacional de Educação. Parecer CNE/CES no 564/2015. 2015 b. Disponível em: http://www.abed.org.br/arquivos/parecer_cne_ces_564_15.pdf. Acesso em: nov. 2017.

BRASIL. Ministério da Educação (MEC). 2017. Disponível em: http://emec.mec.gov.br/ Acesso em: abr./ ago. 2017.

COSTA, L. P. da. Algumas considerações acerca da didática e da educação matemática na educação básica. JORNADA DE DIDÁTICA - O ENSINO COMO FOCO, 1., e FÓRUM DE PROFESSORES DE DIDÁTICA DO ESTADO DO PARANÁ, 1., 2013. Maringá/PR. Anais [...]. UEM, Maringá/PR, 2013.

ESPINOSA, A. J. Didáctica de la matemática, educación matemática e investigación. Revista Ciências em Foco, v. 1, n. 4, 2011. Disponível em: http://ojs.fe.unicamp.br/index.php/cef/article/view/4562. Acesso em: 10 dez. 2017.

FIGUEIREDO, S. A.; COSTA, N. M. L. Prática como componente curricular: uma investigação na licenciatura em matemática. In: LOPES, C. E.; TRALDI, A.; FERREIRA, A. C. (org.). A formação do professor que ensina matemática: aprendizagem docente e políticas públicas. Campinas, SP: Mercado da Letras, 2015. p. 109146.

HARNECKER, M. Os conceitos elementares do materialismo histórico. 2. ed. São Paulo, SP: Global Editora, 1983.

LIBÂNEO, J. C. Didática. 2. ed. São Paulo: Cortez, 2013. 


\section{\& Contexto}

Claudia Maria Witt - Maria Lucia Panossian

NACARATO, A. M.; PAIVA, M. A. V. A formação do professor que ensina matemática: estudos e perspectivas a partir das investigações realizadas pelos pesquisadores do GT7 da SBEM. In: NACARATO, Adair Mendes; PAIVA, Maria Auxiliadora Vilela (org.). A formação do professor que ensina matemática: perspectivas e pesquisas. 3. ed. Belo Horizonte: Autêntica, 2013. p. 7-26.

PESCE, L. O educador em foco: um olhar sobre as políticas de formação docente na modalidade de educação a distância. In: FELDMANN, Marina Graziela (org.). Formação de professores e escola na contemporaneidade. São Paulo: Editora Senac São Paulo, 2009. p. 133-153.

VARIZO, Z. da C. M. Os caminhos da didática e sua relação com a formação de professores de matemática. In: NACARATO, A. M.; PAIVA, M. A. V. A formação do professor que ensina matemática: perspectivas e pesquisas. Belo Horizonte: Autêntica, 2013. p. 43-59. 